\title{
Pesquisa entomológica das espécies de triatomíneos encontradas na zona rural do município de Caruaru, estado de Pernambuco, Brasil, de 2011 a 2012
}

\section{Entomological research of triatomine species found in the rural area of Caruaru, Pernambuco State, Brazil, from 2011 to 2012}

Aline Danielle Santa Cruz de Farias ${ }^{1,2}$ (D), Zaíra Figueiredo Alves ${ }^{1,2}$ (D), Maria Beatriz Araújo Silva ${ }^{1,2}$ iD, Carolina Branco Dale Coutinho3 ${ }^{\text {(iD), Dayse da Silva Rocha }}{ }^{3}$ iD

Universidade de Pernambuco, Faculdade de Enfermagem, Recife, Pernambuco, Brasil

2 Laboratório Central de Saúde Pública "Dr. Milton Bezerra Sobral", Pernambuco, Brasil

${ }^{3}$ Instituto Oswaldo Cruz, Laboratório Nacional e Internacional de Referência em Taxonomia de Triatomíneos, Rio de Janeiro, Rio de Janeiro, Brasil

\begin{abstract}
RESUMO
OBJETIVO: Descrever a presença de triatomíneos na área rural do município de Caruaru, estado de Pernambuco, Brasil, durante os anos de 2011 e 2012, apontando áreas de risco para a doença de Chagas, com base nos registros das principais espécies de triatomíneos. MATERIAIS E MÉTODOS: Os dados sobre os triatomíneos, obtidos no banco de dados do Departamento de Controle de Vetores de Caruaru, foram: número de residências, presença de anexos nas casas, número de habitantes, tipo de moradia, presença de exemplares de triatomíneos, espécies identificadas e localização das moradias. RESULTADOS: Do total de 888 casas estudadas, 85,4\% (758) eram construções de alvenaria com reboco, todas cobertas com teto de telhas, a maioria $(34,1 \% ; 303)$ tinha apenas um anexo e eram habitadas por 2.236 pessoas. Nas 75 (8,4\%) casas onde foram encontrados triatomíneos (41 com anexos e 34 sem anexos), distribuídas em 32 localidades da zona rural de Caruaru, havia 211 (9,4\%) habitantes. As espécies mais comuns encontradas nas residências foram Triatoma brasiliensis (26,8\%), Panstrongylus lutzi (26,8\%) e Triatoma pseudomaculata (12,2\%). CONCLUSÃO: A alteração na distribuição das espécies, na área de estudo, demonstrou a adaptabilidade desses vetores à ação antrópica, reforçando a necessidade da elaboração de políticas educativas e do monitoramento constante nas áreas afetadas, visando à interrupção do ciclo de transmissão do Trypanosoma cruzi.
\end{abstract}

Palavras-chave: Doença de Chagas; Vetores; Triatominae.

\begin{abstract}
OBJECTIVE: To describe the occurrence of triatomines in the rural area of Caruaru City, Pernambuco State, Brazil, from 2011 to 2012, indicating areas of risk for Chagas disease, based on the records of the main triatomine species. MATERIALS AND METHODS: Data about triatomines obtained from Caruaru Vector Control Department database were: number of residences, houses with annexes, number of inhabitants, type of house, occurrence of triatomines, identified species, and location of houses. RESULTS: Of the total of 888 houses analyzed, $85.4 \%$ (758) were plastered masonry constructions all covered with roof tiles, most of them $(34.1 \%, 303)$ with only one annex and inhabited by 2,236 people. In the 75 (8.4\%) houses where triatomines were found (41 with annexes and 34 without annexes), distributed in 32 localities of the rural area of Caruaru, there were 211 (9.4\%) inhabitants. The most common species found in the residences were Triatoma brasiliensis (26.8\%), Panstrongylus lutzi (26.8\%), and Triatoma pseudomaculata (12.2\%). CONCLUSION: The alteration in the distribution of the species, in the study area, demonstrated the adaptability of these vectors to anthropic action, reinforcing the necessity of educational policies and constant monitoring of the affected areas, aiming to interrupt the transmission cycle of Trypanosoma cruzi.
\end{abstract}

Keywords: Chagas Disease, Vectors, Triatominae.

\footnotetext{
Correspondência / Correspondence:

Maria Beatriz Araújo Silva

Universidade de Pernambuco, Faculdade de Enfermagem

Rua Arnóbio Marques, 310. Bairro: Santo Amaro. CEP: 50100-130 - Recife, Pernambuco, Brasil

E-mail: silvamba@yahoo.com.br
} 


\section{INTRODUÇÃO}

A doença de Chagas é um agravo que ocorre somente nas Américas, sendo causada pelo Trypanosoma cruzi (Chagas, 1909), um protozoário flagelado da ordem Trypanosomatida, família Trypanosomatidae. Os triatomíneos são os vetores da doença de Chagas, transmitindo o parasita por meio de suas fezes contaminadas. Além da transmissão vetorial, podem ocorrer também transmissões por via oral, transfusão sanguínea, transplantes de órgãos, acidentes de laboratório, além de iá terem sido registrados casos de transmissão placentária e pelo leite materno'.

Atualmente a doença de Chagas apresenta diminuição de casos registrados, como resultado das medidas de controle e das melhorias das habitações rurais em algumas regiões do Brasil. Com o controle da transmissão vetorial pelo Triatoma infestans (Klug in Meyen, 1834) no início da década de 1990, espécies que eram consideradas secundárias passaram a ocupar lugar de destaque na epidemiologia da doença, entre elas o Triatoma brasiliensis Neiva, 1911, O Triatoma pseudomaculata Correa and Espínola, 1964 e o Panstrongylus lutzi (Neiva and Pinto, 1923)².

A Região Nordeste do Brasil é considerada uma área de grande importância epidemiológica para a doença de Chagas. Em inquéritos nacionais, essa Região apresenta o maior número de pessoas infectadas por T. cruzi, com índices elevados de infestação por triatomíneos ${ }^{3,4,5}$.

Estima-se que aproximadamente 4,6 milhões de pessoas estejam infectadas pelo T. cruzi no Brasil; entretanto, esse número pode ser ainda maior, uma vez que apenas os casos agudos são de notificação compulsória. Dados do Ministério da Saúde mostram que foram registrados, no período de 2001 a 2014, um total de 3.827 casos, sendo 1.625 (42,5\%) na Região Nordeste, seguidos pelas Regiões Norte (1.580; 41,3\%), Sul $(291 ; 7,6 \%)$, Sudeste $(222 ; 5,8 \%)$ e Centro-Oeste $(109 ; 2,8 \%)^{6}$.
Na Região Nordeste, foi registrada a ocorrência de 29 espécies de triatomíneos. Segundo o Programa de Controle de Doença de Chagas, as espécies mais capturadas são: T. brasiliensis e T. pseudomaculata, sendo o T. brasiliensis encontrado em todos os Estados da Região $0^{7,8,9,10}$.

O município de Caruaru, no estado de Pernambuco, foi escolhido para este estudo em virtude do alto índice de triatomíneos encontrados em coletas realizadas pela IV Gerência Regional de Saúde (GERES) do Estado. O presente trabalho descreve a presença de triatomíneos na área rural de Caruaru, durante os anos de 2011 e 2012, definindo as áreas de risco para a doença de Chagas com base nos registros das principais espécies.

\section{MATERIAIS E MÉTODOS}

O município de Caruaru (S816 $53^{\prime \prime}$, W3558'25") está inserido na unidade geoambiental do Planalto da Borborema e incluído na área geográfica de abrangência do semiárido brasileiro. Em 2010, tinha uma população de 314.951 habitantes, vivendo em uma área territorial de $920.606 \mathrm{~km}^{2}$, cuja densidade demográfica correspondia a 342,07 hab. $/ \mathrm{km}^{2}$, e está distribuída em quatro distritos ${ }^{4}$ (Figura 1).

A área de investigação foi a zona rural de Caruaru, com população de 35.323 habitantes $(11,2 \%)$, onde os agentes de saúde municipais realizam monitoramento e coletas de triatomíneos. Os dados utilizados são provenientes do banco de dados do Departamento de Controle de Vetores da cidade e correspondem aos anos de 2011 e 2012. Os principais indicadores avaliados foram: pertencer à zona rural (sítios, povoados ou fazendas da localidade), número de residentes na casa, presença de anexos nas casas, tipo da moradia, presença ou ausência de exemplares de triatomíneos.

As moradias foram classificadas de acordo com o tipo de material com que foram construídas: alvenaria com reboco, alvenaria sem reboco, barro com reboco e barro sem reboco.

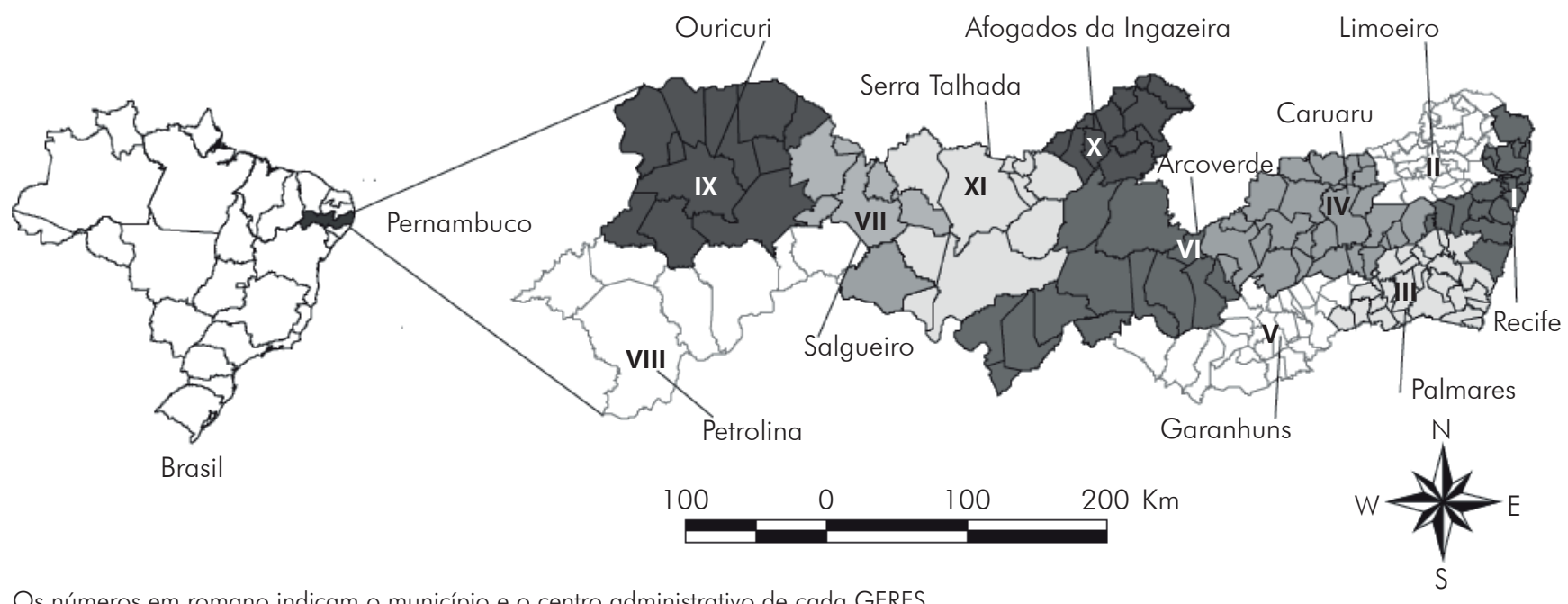

Figura 1 - Localização do estado de Pernambuco no Brasil e suas 11 GERES 
Os insetos foram capturados manualmente por técnicos de endemias do Município, utilizando pinças e lanternas para a coleta, além da aplicação de líquido desalojante (Pirisa 2\%) para a retirada dos insetos de frestas profundas. Os exemplares coletados foram encaminhados para o Laboratório Municipal de Entomologia da IV GERES, sendo posteriormente enviados ao Laboratório de Endemias do Laboratório Central de Saúde Pública de Pernambuco para a identificação das espécies. A coleta de material para - exame parasitológico foi feita pela compressão abdominal do espécime e posterior exame de suas fezes em microscópio óptico (400x).

As coletas manuais foram realizadas no intradomicilio e em todos os anexos que constituem o peridomićlio, como galinheiros, chiqueiros, pequenos galpões que serviam de armazenamento de materiais diversos e demais estruturas que, mesmo precárias, poderiam servir de abrigo para os insetos.

Para as análises estatísticas descritiva e inferencial, foi utilizado o programa SPSS (Statistical Package for the Social Sciences, v. 17), no qual foram calculadas as distribuições absolutas, os percentuais uni e bivariados e aplicado o teste qui-quadrado de Pearson. Para as decisões dos testes estatísticos, foi adotado o intervalo de confiança de $95 \%$.

\section{RESULTADOS}

Foram avaliados dados de 888 residências da zona rural de Caruaru. Dessas, 57,1\% (507) tinham anexos, sendo a maioria, 34,1\% (303), com apenas um anexo. $O$ tipo de parede mais encontrado foi o de alvenaria com reboco, representando $85,4 \%$ (758) das residências. Todas eram cobertas com teto de telha. Em relação ao número de habitantes, 24,7\% (219) das residências estavam desocupadas e, dentre as ocupadas, a maioria possuía dois habitantes (16,4\%; 146) (Tabela 1). As 888 residências apresentaram um total de 2.236 habitantes, dos quais 9,4\% (211) residiam em casas onde foram encontrados espécimes de triatomíneos e, por isso, foram classificados como expostos.

Também foi analisada a associação entre a ocorrência de triatomíneos e a presença de anexos e habitantes expostos. Foi detectada associação significativa da presença de triatomíneos com a presença de habitantes expostos e, para essa variável, destaca-se que o percentual de residências com triatomíneos foi mais elevado nas residências com habitantes que nas sem habitantes, $10,0 \%$ e 3,7\%, respectivamente (Tabela 2 ).

De todas as habitações investigadas, 75 (8,4\%) foram positivas para a presença de triatomíneos (41 com anexos e 34 sem anexos), distribuídas em 32 localidades dos Distritos de Carapotós, Gonçalves Ferreira e Lajedo do Cedro, todos localizados na zona rural: sete casas em Carneirinho; seis em Malhada B. Queimada; cinco em Riacho Doce, Baixo II e Lajes I, cada; quatro em Dois Riachos e Cachoeira Seca, cada; três em Caldeirão, Mocós e Queimados, cada; dois em Jacaré Grande, Xicurú, Laje, Reinado, Carapotós, Itaúna, Serrote dos Bois e Serrote Preto, cada; um em Pítia do Capim, Poço Velho, Salgadinho, Cipó, Santa Maria, Lagoa do Exu, Olho D'Água do Felix, Caldas, Pingueira, Fundão, Serra do Rodrigues, Normandia, Serra de São Francisco e Muniz, cada.

Tabela 1 - Características das residências pesquisadas da zona rural de Carvaru, estado de Pernambuco, Brasil, 2011 e 2012

\begin{tabular}{lcc}
\hline \multicolumn{1}{c}{ Variáveis } & $N=888$ & $\%$ \\
\hline Anexos na residência & 507 & 57,1 \\
Sim & 381 & 42,9 \\
Não & & \\
Número de anexos na residência & 381 & 42,9 \\
Nenhum & 303 & 34,1 \\
Um & 127 & 14,3 \\
Dois & 77 & 8,7 \\
Três ou mais & & \\
Tipo de parede da residência & 758 & 85,4 \\
Alvenaria com reboco & 107 & 12,0 \\
Alvenaria sem reboco & 18 & 2,0 \\
Barro com reboco & 5 & 0,6 \\
Barro sem reboco & & \\
Tipo de teto da residência & 888 & 100,0 \\
Telha & & \\
Número de habitantes por residência & 219 & 24,7 \\
Nenhum & 94 & 10,6 \\
Um & 146 & 16,4 \\
Dois & 144 & 16,2 \\
Três & 139 & 15,7 \\
Quatro & 87 & 9,8 \\
Cinco & 59 & 6,6 \\
Seis ou mais & &
\end{tabular}


Tabela 2 - Variação da ocorrência de triatomíneos, segundo a presença de anexos e habitantes, em residências da zona rural de Caruaru, estado de Pernambuco, Brasil, 2011 e 2012

\begin{tabular}{|c|c|c|c|c|c|c|c|}
\hline \multirow{3}{*}{ Variáveis } & \multicolumn{4}{|c|}{ Triatomíneos } & \multirow{2}{*}{\multicolumn{2}{|c|}{ Total }} & \multirow{3}{*}{ p } \\
\hline & \multicolumn{2}{|c|}{ Presentes } & \multicolumn{2}{|c|}{ Ausentes } & & & \\
\hline & $\mathrm{N}$ & $\%$ & $\mathrm{~N}$ & $\%$ & $\mathrm{~N}$ & $\%$ & \\
\hline Total de residências & 75 & 8,4 & 813 & 91,6 & 888 & 100,0 & \\
\hline \multicolumn{8}{|l|}{ Residências com anexos } \\
\hline Sim & 41 & 8,1 & 466 & 91,9 & 507 & 57,1 & $\mathrm{p}^{+}=0,657$ \\
\hline Não & 34 & 8,9 & 347 & 91,1 & 381 & 42,9 & \\
\hline \multicolumn{8}{|l|}{ Habitantes nas residências } \\
\hline Sim & 67 & 10,0 & 602 & 90,0 & 669 & 75,3 & $p^{\ddagger}=0,003^{*}$ \\
\hline Não & 8 & 3,7 & 211 & 96,3 & 219 & 24,7 & \\
\hline
\end{tabular}

* Associação significativa de 5,0\%; † Teste qui-quadrado de Pearson; ${ }^{\ddagger}$ Teste exato de Fisher.

Foram encontrados 82 exemplares de triatomíneos nas 75 casas infestadas, dos quais somente 54 (65,8\%) foram identificados: P. lutzi $(22 / 26,8 \%), T$. brasiliensis $(22 / 26,8 \%)$ e T. pseudomaculata $(10 / 12,2 \%)$ (Tabelas 3 e 4).

\section{DISCUSSÃO}

Existem fatores que podem favorecer a disseminação da doença de Chagas, como a baixa situação socioeconômica, que se reflete nas péssimas condições das habitações humanas, e a ocorrência de espécies de triatomíneos com importante potencial de domiciliação e capacidade vetorial 11,12,13. Geralmente os domicílios são construídos de forma precária: paredes de pau a pique, barreadas e com telhado de sapé, tábuas de madeira e alvenaria sem reboco, facilitando a adaptação dos insetos a esses habitats artificiais ${ }^{14}$.

No presente estudo, foi evidenciada uma modificação na estrutura dos domicílios, já que 85,4\% das residências eram de alvenaria com reboco e todas possuíam telha, demonstrando uma adaptação do vetor às residências de melhor estrutura. Esses resultados sugerem que as moradias de alvenaria, que antes eram consideradas barreiras para o encontro de insetos no intradomicílio, precisam apresentar outras estruturas que possam impedir a entrada dos triatomíneos, como telas nas janelas e forro no telhado, para dificultar a penetração dos vetores, principalmente nos períodos noturnos, já que a iluminação artificial pode atuar como fonte de atração $^{15}$.

Os dados mostraram ainda que, das 888 casas analisadas, 75 (8,4\%) apresentavam triatomíneos; dessas, 41 (54,7\%) possuíam anexo e 34 (45,3\%) não, as quais tinham um total de 211 habitantes expostos ao vetor. Os anexos, normalmente de estrutura precária, aumentam a oferta de nichos para serem ocupados por insetos e, assim, expõem os residentes a vetores de doenças.

Tabela 3 - Quantidade de triatomíneos encontrados nas residências analisadas da zona rural de Caruaru, estado de Pernambuco, Brasil, 2011 e 2012

\begin{tabular}{lcr}
\hline \multicolumn{1}{c}{ Variáveis } & Residências & $\%$ \\
\hline Número de triatomíneos encontrados por residência & 39 & 52,0 \\
Um & 6 & 8,0 \\
Dois & 3 & 4,0 \\
Três & 27 & 36,0 \\
Não informado & 75 & 100,0 \\
\hline Total
\end{tabular}

Tabela 4 - Espécies de triatomíneos encontrados nas residências analisadas da zona rural de Caruaru, estado de Pernambuco, Brasil, 2011 e 2012

\begin{tabular}{lcr}
\hline \multicolumn{1}{c}{ Variáveis } & Residências & $\%$ \\
\hline Número de triatomíneos encontrados por residência & & \\
Triatoma brasiliensis & 22 & 26,8 \\
Panstrongylus lutzi & 22 & 26,8 \\
Triatoma pseudomaculata & 10 & 12,2 \\
Não informado & 28 & 34,2 \\
\hline Total & 82 & 100,0 \\
\hline
\end{tabular}


Nas moradias onde foram encontrados os triatomíneos, observou-se que as únicas espécies encontradas foram T. brasiliensis (26,8\%), P. lutzi $(26,8 \%)$ e T. pseudomaculata $(12,2 \%)$, corroborando os estudos anteriores que mostraram essas espécies como as mais prevalentes na área de abrangência da IV GERES de Pernambuco. Silva et al. ${ }^{11}$ descreveram T. pseudomaculata, T. brasiliensis e P. lutzi como os principais vetores no Nordeste do Brasil, e que - T. brasiliensis está amplamente distribuído em Pernambuco, sendo essas as principais espécies capturadas entre 2006 e 2007. No presente estudo, P. lutzi aparece em igual proporção ao T. brasiliensis, $26,8 \%$, mostrando seu crescimento no contexto histórico dos vetores no Estado, já que, no estudo desenvolvido por Silva et al. ${ }^{14}$, a espécie $P$. lutzi foi a terceira mais abundante. Esse fato altera o quadro observado por diversos autores $16,17,18,19$, que destacaram 0 T. brasiliensis e o T. pseudomaculata como as principais espécies capturadas em Pernambuco.

Tais informações demonstram a importância da realização de inquéritos entomológicos, para verificar a adaptação das espécies de triatomíneos em áreas rurais que sofrem constantes modificações ${ }^{16,17,18,19}$, como, por exemplo, o desmatamento para a criação de áreas de lavoura e/ou moradia. Nesse contexto, é primordial que seja determinada a taxa de positividade para a infecção por T. cruzi dos exemplares das espécies de triatomíneos encontradas, para assim poder verificar o papel de cada uma na transmissão da doença de Chagas.

A Região Nordeste apresenta um alto percentual de transmissão vetorial domiciliar que, mesmo com a redução dos focos de $T$. infestans, vem sendo perpetuado devido ao persistente encontro de espécies nativas $^{20,21,22}$. Na Região Norte, foi observado um aumento no número de casos confirmados por meio da transmissão oral e, em direção contrária, as Regiões Sudeste, Sul e Centro-Oeste vêm conseguindo diminuir, ao longo dos anos, os casos de mortalidade por doença de Chagas, reflexo de políticas para o tratamento de pacientes chagásicos e, principalmente, de controle dos vetores ${ }^{23}$.

\section{CONCLUSÃO}

Foi demonstrada a alteração na distribuição das espécies de triatomíneos na área estudada quanto à frequência, pois o $P$. lutzi foi tão frequente quanto o T. brasiliensis, demonstrando a capacidade adaptativa de espécies de triatomíneos à ação antrópica.

Portanto, os resultados deste estudo demostraram a necessidade do aumento e da regularidade das ações de vigilância entomológica em Caruaru, juntamente com a elaboração de políticas educativas para as populações locais e o tratamento das áreas afetadas, visando à interrupção do ciclo de transmissão do T. cruzi aos humanos.

\section{CONFLITOS DE INTERESSE}

Os autores declaram que não houve conflitos de interesse.

\section{CONTRIBUIÇÃO DOS AUTORES}

Todos os autores contribuíram com a idealização do estudo, a análise e a interpretação dos dados e com a redação do manuscrito, aprovando a versão final publicada. Declaram-se responsáveis pelo conteúdo integral do artigo, garantindo sua precisão e integridade.

\section{REFERÊNCIAS}

1 Tatto E, Padilha EM, Fernandes SB. Aspectos epidemiológicos, diagnóstico e tratamento: guia de consulta rápida para profissionais de saúde. Rev Pat Trop. 2007;36:1-32.

2 Silveira AC, Martins E. Histórico do controle da transmissão vetorial e situação epidemiológica atual. In: Galvão C, organizador.Vetores da doença de Chagas no Brasil. Curitiba: Sociedade Brasileira de Zoologia; 2014. p. 10-25. (Série Zoologia: guias e manuais de identificação).

3 Briceño-León R, Méndez Galván J. The social determinants of Chagas disease and the transformations of Latin America. Mem Inst Oswaldo Cruz. 2007 Oct;30(102 Suppl 1):109-12.

4 Instituto Brasileiro de Geografia e Estatística. Sinopse do senso demográfico 2010: Pernambuco [Internet]. Rio de Janeiro: IBGE; 2010. [citado 2018 dez 23]. Disponível em: https://censo2010. ibge.gov.br/sinopse/index. php?dados $=29 \& u f=26$.

5 Dias JCP, Machado EMM, Fernandes AL, Vinhaes MC. Esboço geral e perspectivas da doença de Chagas no Nordeste do Brasil. Cad Saude Publica. 2000 jan;16 supl 2:13-34.

6 Brasileiro MR. Esquisse of the epidemiological scenario of Chagas disease in Brasil. Immunol Case Rep J. 2017;1 (2):17-9.

7 Galvão C, organizador. Vetores da doença de Chagas no Brasil. Curitiba: Sociedade Brasileira de Zoologia; 2014. (Série Zoologia: guias e manuais de identificação).

8 Mota JC, Campos MR, Schramm JMA, Costa MFS. Estimativa de taxa de mortalidade e taxa de incidência de sequelas cardíacas e digestivas por doença de Chagas no Brasil. Epidemiol Serv Saude. 2008 out-dez;23(4):711-20.

9 Fundação Nacional de Saúde (BR). Elaboração de projeto de melhoria habitacional para o controle da doença de Chagas: orientações técnicas. Brasília: Funasa; c2004. 
10 Costa J, Almeida CE, Dotson EM, Lins A, Vinhaes $M$, Silveira $A C$, et al. The epidemiologic importance of Triatoma brasiliensis as a Chagas disease vector in Brazil: a revision of domiciliary captures during 1993-1999. Mem Inst Oswaldo Cruz. 2003 Jun;98(4):443-9.

11 Silva MBA, Barreto AVMS, Silva HA, Galvão C, Rocha D, Jurberg J, et al. Triatomíneos sinantrópicos (Hemiptera, Reduviidae) no Estado de Pernambuco, Brasil: distribuição geográfica e índices de infecção natural por Trypanosoma entre 2006 e 2007. Rev Soc Bras Med Trop. 2012 jan-feb;45(1):60-5.

12 Silva MBA, Menezes KR, Siqueira AM, Balbino $V Q$, Lorosa ES, Farias MCG, et al. Importância da distribuição geográfica dos vetores da doença de Chagas em Pernambuco, Brasil, em 2012. Rev Patol Trop. 2015 abr-jun;44(2):195-206.

13 Silva MBA, Menezes KR, Farias MCG, Souza Filho IB, Jurberg J. Vigilância entomológica dos vetores da doença de Chagas no agreste pernambucano. Rev Saude Desenvolv. 2017 abr-jun;1 1 (7):229-44.

14 Silva MBA, Menezes KR, Farias MCG, Andrade MS, Victor CCA, Lorosa ES, et al. Description of the feeding preferences of triatominae in the Chagas disease surveillance study for the State of Pernambuco, Brazil (Hemiptera: Reduviidae). Rev Soc Bras Med Trop. 2017 Jul-Aug;50(4):543-6.

15 Dias JCP. Problemas e possibilidades de participação comunitária no controle das grandes endemias no Brasil. Cad Saude Publica. 1998;14 supl 2:S19-37.

16 Costa J, Almeida JR, Britto C, Duarte R, Marchon-Silva V, Pacheco RS. Ecotopes, natural infection and trophic resources of Triatoma brasiliensis (Hemiptera, Reduviidae, Triatominae). Mem Inst Oswaldo Cruz. 1998 Jan-Feb;93(1):7-13.
17 Dias-Lima AG, Menezes D, Sherlock I, Noireau F. Wild habitat and related fauna of Panstrongylus lutzi (Reduviidae:Triatominae). J Med Entomol. 2003 Nov;40(6):989-90.

18 Gurgel-Gonçalves R, Cuba CAC. Predicting the potential geographical distribution of Rhodnius neglectus (Hemiptera, Reduviidae) based on ecological niche modeling. J Med Entomol. 2009 Jul;46(4):952-60.

19 Gurgel-Gonçalves R, Galvão C, Costa J, Peterson AT. Geographic distribution of Chagas disease vectors in Brazil based on ecological niche modeling. J Trop Med. 2012;2012:705326.

20 Lima MM, Sarquis $O$. Is Rhodnius nasutus (Hemiptera: Reduviidae) changing its habitat as a consequence of human activity? Parasitol Res. 2008 Mar; 102(4):797-800.

21 Dias JCP. Southern Cone Initiative for the elimination of domestic populations of Triatoma infestans and the interruption of transfusional Chagas disease. Historical aspects, present situation, and perspectives. Mem Inst Oswaldo Cruz. 2007 Oct;102 Suppl 1:11-8.

22 Silveira AC. Os novos desafios e perspectivas futuras do controle. Rev Soc Bras Med Trop. $2011 ; 44$ supl 2:122-4

23 Ministério da Saúde (BR) Secretaria de Vigilância em Saúde. Doença de Chagas aguda no Brasil: série histórica de 2000 a 2013. Bol Epidemiol. 2015;46(21): $1-9$. 